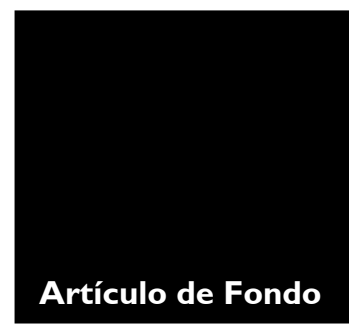

\title{
PERSPECTIVAS LATINOAMERICANAS PARA EL ESTUDIO DE LOS SERVICIOS URBANOS
}

\section{Pedro Pírez}

Investigador principal del Conicet con sede en el Instituto de Estudios de América Latina y el Caribe de la Facultad de Ciencias Sociales de la Universidad de Buenos Aires.

ISNN 1666-6I86.Volumen I4 N I4 (Junio de 20I3) pp. I73-192 - Recibido: 05-03-20I3 Aceptado: 10-04-20I3 


\section{Resumen}

Los servicios urbanos son un componente esencial de la urbanización que define el suelo urbanizado y permite el funcionamiento cotidiano del conjunto de los soportes físicos. Este artículo propone construir un marco conceptual para dar cuenta de los servicios a partir de los estudios urbanos de la región. Identifica tres perspectivas: estudios globales de la urbanización latinoamericana que atienden a la reproducción de las formaciones sociales capitalistas; análisis de los procesos de la urbanización latinoamericana, en la producción de la urbanización popular, y trabajos relativos a la gestión urbana. Esas perspectivas diferencian dos momentos del desarrollo: desde los años 40 del siglo XX una fase de industrialización sustitutiva de importaciones y políticas económicas keynesianas y de bienestar y, luego de la crisis de los años 70 y 80, de reestructuración neoliberal. Con base en esas presentaciones, presenta conclusiones que intentan sistematizar los elementos del análisis.

\section{Palabras clave}

Servicios urbanos, estudios urbanos latinoamericanos, urbanización popular, gestión urbana, desmercantilización.

\section{$\underline{\text { Abstract }}$}

Urban services are an essential component of urbanization which create urban land and allow for the daily operation of all physical supports. This paper uses the contributions of Latin-American urban studies, to propose a conceptual framework to explain the nature of urban services. It identifies three perspectives: global studies of Latin-American urbanization that analyze the process of reproduction of capitalist social formations; the urbanization process and the production of popular urbanization projects ; and studies on urban management. These perspectives distinguish two periods of the development process: from the forties to the sixties of the twentieth century, a phase of import substitution, Keynesian economic policies and welfare; and, after the crisis of the seventies and eighties, neoliberal restructuring. Based on these studies, the conclusions try to present elements for systematic analysis.

\section{Keywords}

Urban Services, Latin-American Urban Studies, Popular Urbanization, Urban Management, Decommodification. 


\section{INTRODUCCIÓN}

Los servicios urbanos son un componente esencial de los procesos de urbanización. Un territorio urbanizado cuenta con un conjunto de bienes físicos, además del suelo, y de servicios que permiten (sustentan) el asentamiento social aglomerado. Esos servicios pueden clasificarse en dos grupos fundamentales: de redes o de infraestructura y de mantenimiento. Los primeros se sustentan en una infraestructura física que permite la prestación del servicio. Por ejemplo, las redes de ductos para la distribución del agua o del gas, o las redes de cables para la distribución de la electricidad. Es de sentido común que esos componentes, tanto los físicos como los servicios, definen el suelo urbanizado. Los segundos permiten el funcionamiento cotidiano del conjunto de los soportes físicos, como es el caso del tratamiento de los residuos sólidos urbanos, o el mantenimiento propiamente dicho de las vialidades. En este artículo revisamos la investigación que se ha realizado en América Latina sobre los servicios urbanos, para construir un marco analítico que permita dar cuenta de ellos en estas sociedades. Pretendemos con esto contribuir a un tratamiento científico de los servicios urbanos dentro de los procesos de urbanización en América Latina, a partir de los aportes de los estudios urbanos de la región. Para ello identificamos tres perspectivas que permiten sustentar esa tarea. Los estudios globales de la urbanización latinoamericana que atienden a la reproducción de las formaciones sociales capitalistas en perspectivas de economía política; el análisis de los procesos diferenciales de la urbanización latinoamericana, en lo que fue llamado la producción del hábitat popular o bien urbanización popular, y los trabajos relativos a la gestión urbana.

Esas tres perspectivas son parte de los estudios urbanos de los servicios urbanos. Esto es, análisis que enfatizan el lugar de esos servicios en los procesos de producción, configuración y funcionamiento de las ciudades o áreas urbanas. Quedan fuera, por ejemplo, miradas sectoriales, sean en perspectivas técnicas, económicas o políticas. ${ }^{1}$

Los trabajos realizados en aquellas tres perspectivas tendieron a diferenciar dos momentos del desarrollo del capitalismo, tanto internacionalmente como en la región. El primero, desde los años 40 del siglo XX, se prolonga aproximadamente hasta fines de los años 70, y se caracteriza por la industrialización sustitutiva de importaciones y la aplicación de políticas económicas keynesianas y de bienestar ${ }^{2}$; el segundo, perceptible en la región a partir de la crisis de los años 70 y 80 , es parte de la reestructuración internacional orientada por concepciones neoliberales. $\overline{\text { 1- Por ejemplo, los trabajos }}$ del grupo de FLACSO Buenos Aires, dirigidos por Daniel Aires, dirigidos por Daniel
Aspiazu (2002), vinculados con las privatizaciones de los $S U$ en sus dimensiones fundamentalmente económicas, o los de Murillo (2004) en miradas políticas sobre esos mismos fenómenos.

2- Debe recordarse que en los países centroamericanos y en alounos países andinos eso procesos fueron posteriores $y$ procesos fueron
más débiles aun. 
A continuación presentamos cada una de esas tres perspectivas, relacionándolas con los dos momentos que hemos señalado. Terminamos con unas conclusiones que ofrecen un intento de sistematizar los elementos del análisis.

\section{LA ECONOMÍA POLÍTICA DE LOS SERVICIOS URBANOS}

El desarrollo de las relaciones capitalistas implica la socialización de las fuerzas productivas. Para ello tiene un papel relevante el conjunto de "consumos colectivos" que se integran por valores de uso que, siendo "indispensables para la acumulación del capital en general", son "dificiles de suministrar por el capital individual". Se trata de "los servicios públicos, la vialidad y los espacios colectivos, los servicios de salud, de educación y la vivienda para sectores populares, etc." (JARAMILlo, 1988: 15 y 16).

La ciudad es uno de los mecanismos de la cooperación que permite "la concentración fisica y la simultaneidad de los procesos" que potencia la capacidad productiva del trabajo, el entrelazamiento de diversos procesos de producción y el acceso a los valores de uso colectivo. Esto es un resultado incierto de las relaciones de múltiples intereses, muchas veces contradictorios (JARAMILLO, 1988: 19) en el mercado. Sin embargo, esos valores de uso indispensables para la acumulación en su conjunto no pueden ser producidos por los capitales individuales: la escala requerida lo hace muy difícil, no es posible dividir el consumo colectivo, falta demanda solvente o el período de rotación del capital es muy largo. $\mathrm{Si}$ esos consumos colectivos circulasen mercantilmente, la tasa de ganancia individual “comprometería seriamente la tasa de ganancia global”. (JARAMILLO, 1988: 20).

Esos "límites de la socialización capitalista" disminuyen el espacio de maniobra de los capitales individuales para garantizar la reproducción de sus condiciones de acumulación. Para los sectores populares esto supone el deterioro de consumos esenciales a sus "formas de vida", y genera respuestas políticas, con diferentes movilizaciones, y contradicciones con el capital (JARAMILLO, 1988).

Las proposiciones anteriores son válidas para el "capitalismo central" en una fase particular de su desarrollo, que se configura desde la superación de la economía liberal, por la intervención estatal, y se consolida con el Estado de Bienestar. Se caracterizó por el fordismo en la organización de la producción, el predominio del trabajo asalariado, políticas macroeconómicas keynesianas, y un pacto social de redistribución. Con el predominio del Estado de Bienestar, la superación de aquellas limitaciones asumió dos modalidades fundamentales. Por una parte, diferentes formas de intervención del Estado, que tomó a su cargo la producción de esos valores de uso creando "organismos 'capitalistas desvalo- 
rizados ", que contribuyen a la "reproducción del capital en su conjunto". Por otra parte, intervenciones que crean condiciones para que el capital privado opere donde no podría hacerlo de manera espontánea, aplicando diferentes instrumentos: subsidios, transferencias, concesión de monopolios (JARAMILLO, 1988).

La intervención estatal fue la respuesta más consistente, aunque no la única. Se dieron alternativas privadas (no estatales) no capitalistas (no buscan ganancia), con la pervivencia y aun proliferación de formas de producción más primitivas, como la producción mercantil no capitalista, que buscan su reproducción simple, o modalidades no mercantiles como "el autosuministro y el trabajo doméstico" (JARAMILLO, 1988: 21). ${ }^{3}$

Para poder intervenir, el Estado debe tomar recursos financieros desde las diferentes formas de plusvalía acumulada por el capital o desde los salarios. Este proceso implica una dimensión económica y otra política. Por una parte, existe "un componente de racionalidad económica capitalista", dado que cada capitalista individual se desprende de parte de su ganancia directa para asegurar condiciones generales de su acumulación que no puede producir por sí mismo. La gestión del Estado se orienta así por la "racionalidad capitalista" eligiendo sus acciones de manera que "tengan el impacto más favorable sobre la acumulación de capital". La dimensión política está asociada con la legitimación de la dominación, al garantizar condiciones de distribución más allá del mercado. Sin embargo, como cada capitalista individual procura que los costos no recaigan sobre él, es necesario el ejercicio de cierta coerción, que depende de la relación de fuerzas políticas y de la capacidad estatal para hacerse de recursos. Por otra parte, es necesario, también, dar respuesta a conflictos concretos. La movilización popular puede obtener concesiones para el suministro de bienes de consumo colectivo, tendiendo a superar los límites estructurales de la acción estatal (JARAMILLO, 1988: 23-27). La fuente de los fondos que utiliza el Estado, la plusvalía acumulada por el capital o los salarios de los trabajadores, califica la significación de las acciones estatales. En el primer caso podrá tratarse de redistribuciones verticales, mientras que en el segundo, horizontales. Estas no modifican las condiciones de concentración social de los recursos.

En las sociedades latinoamericanas, la urbanización fue el resultado de su incorporación en la división internacional del trabajo, insertándose en la industrialización que se desarrollaba en otros territorios (Europa y EE UU). De allí la falta de correlación entre indicadores de urbanización e industrialización.

La acumulación de capital se estructuró de manera subordinada, transfiriendo buena parte del excedente a las economías centrales. Una importante cantidad de población queda
3-Estas alternativas no sirven para la producción de valores de uso que "requieren técnicas sofisticadas", o lo hacen cas sofisticadas", o lo hacen
con poca eficiencia y mala calidad. 
fuera del mercado de fuerza de trabajo o es incorporada en forma precaria, y opera como "ejército de reserva" que contribuye a mantener bajos los salarios. Los estados tienen una limitada capacidad de captación fiscal, asociada a la acumulación subordinada y a la debilidad política frente a los sectores económicamente poderosos, muchos de ellos de base internacional.

Los estados latinoamericanos intervienen en la producción de servicios urbanos desde inicios del siglo XX como parte de un intento de responder a las necesidades de la acumulación económica y al fortalecimiento de la legitimidad política, garantizando a los trabajadores, y aun a la población no incluida en las relaciones salariales, cierto nivel en las condiciones materiales de subsistencia (Pradilla, 2009: 137-138). De todas formas, predominó la debilidad estatal, propia de las condiciones de acumulación capitalista subordinada y de la consecuente "limitación relativa de los recursos que la formación social como un todo puede destinar a la creación de estas condiciones generales de la acumulación, y en particular, a los valores de uso colectivo urbanos" (JARAMILLO, 1988: 28). Esa debilitad se relaciona con las condiciones sociopolíticas predominantes, que afectan la capacidad gubernamental de orientar las tensiones estructurales y reducen el peso específico del Estado frente al capital privado. Aquel tuvo un papel limitado como unificador de los intereses globales de las clases explotadoras y promotor de estrategias de largo plazo, predominando los intereses inmediatos de las fracciones dominantes. De ahí la dificultad para exigir al capital privado parte de sus ganancias para la provisión de consumos colectivos. Los recursos estatales se asignaron predominantemente para crear "valores de uso directamente ligados a la acumulación de capital, y en particular a la producción de mercancías, en detrimento de aquellos ligados con el consumo y, especialmente, con la reconstitución de las capas populares". Complementariamente, esos recursos se concentraron en pocos centros primados, reforzando la desigualdad de los equipamientos y las tendencias a la concentración espacial. Como consecuencia, el Estado descargó sobre los sectores populares la provisión de algunos de esos bienes, en lo que se configuró como urbanización popular (JARAMILLO, 1988: 29 y 31). Si bien predominó la racionalidad económica frente a la política, subordinando la legitimidad, la "descarga" estatal fue acompañada, en gran medida, por relaciones de carácter clientelar.

La crisis de los años 70-80 afectó el desarrollo del capitalismo en la región, con el agotamiento del modelo de acumulación basado en la sustitución de importaciones. Se sufrió, también, el fin del período de rápida acumulación del capitalismo internacional.

Esos cambios limitaron más aun los recursos destinados a la producción de bienes de consumo colectivo, mientras que las necesidades crecían, y se acentuaba la tendencia a 
abaratar la mano de obra. Esa producción se sesgó más en contra de la reproducción de la fuerza de trabajo, incrementando las desigualdades sociales e interregionales y deteriorando el consumo (JARAMILLO, 1988: 34).

La contradicción entre la necesidad de bienes de consumo colectivos y la dificultad para producirlos se mostró como parte de una fase del desarrollo del capitalismo. Como dice CUERVO: "las restricciones técnicas y económicas para la 'rentabilización"', de los medios de consumo colectivo urbanos "estaban históricamente determinadas". Podían entonces cambiar, "en algunos casos profundamente, como efectivamente se ha dado durante los últimos 20 años" (CUERVO, 1997), como parte de un proceso más amplio de reestructuración.

La reestructuración neoliberal del capitalismo a nivel internacional — luego de la detención del proceso de crecimiento con pleno empleo, rentabilidad creciente y estabilidad que caracterizaron a los treinta años posteriores a la segunda posguerra - puede definirse por la apropiación por el capital de una porción cada vez mayor del excedente, negando el acuerdo de bienestar. Combinó aumento de la productividad y nivel de explotación; cambio del modelo de intervención estatal, con énfasis en el dominio político y la acumulación del capital, en detrimento de la legitimación política y la redistribución social. La internacionalización de los procesos económicos contribuyó para incrementar la rentabilidad y abrir mercados para su expansión (CASTELLS, 1995: 52-57). Bastaría resaltar el creciente predominio del sector financiero y la "financierización" de la economía en su conjunto, incluyendo la producción urbana y de los servicios. Esos cambios económicos, sociales y políticos fueron justificados y valorados con el creciente predominio de ideas neoliberales (HARVEY, 2007).

Las transformaciones tecnológicas permitieron que las industrias de telecomunicaciones, energía y servicios urbanos - especialmente agua y recolección y tratamiento de basurassurgiesen como una posibilidad inexplorada de ganancias y de acumulación. Los capitales de los países centrales se abrieron paso en esas actividades utilizando medios como el comercio, la financiación y las concepciones de manejo económico (CUERVo, 1998: 457).

En América Latina la asfixia financiera de los años 80 impidió que sus países se convirtieran en consumidores de esos nuevos circuitos, pero, al mismo tiempo, fortaleció el cambio de las concepciones sobre el papel del Estado y su relación con la economía, dando pie a la influencia de las ideas neoliberales. El norte comenzó a crecer más lentamente y el capital multinacional se orientó hacia las economías del sur (CuERvo, 1998: 457). La caída de las tasas de interés internacionales, el debilitamiento del dólar en América Latina y el 
$\overline{4-\text { También denominada }}$ "urbanización formal".

5- Sobre las lógicas sociales en la producción de la ciudad ver Pírez, 1995. impacto de la reestructuración de la deuda externa aligeraron las cargas financieras de la región y mejoraron las posibilidades de los países de insertarse en los nuevos circuitos comerciales y financieros. Se dieron así las condiciones para la reforma del Estado y de la economía: reducción de aranceles, liberalización financiera y cambiaria, flexibilización laboral y, en particular, privatizaciones.

Los servicios urbanos en la región modificaron su orientación: competencia en lugar de monopolio, autosuficiencia financiera y aplicación de mecanismos de mercado (CoING, 2005: 137). Su producción se orientó a conservar o conquistar clientes sin invertir en las zonas de población de menores recursos, que suelen ser poco rentables. Al mismo tiempo, la competencia eliminó la posibilidad de aplicar subsidios cruzados, ya que los grandes consumidores pueden buscar otro proveedor, negociar con él su tarifa y evadir el sobreprecio (COING, 2005: 147). Las privatizaciones transformaron las relaciones sociales al introducir empresas de gran poder, por lo general extranjeras, con gran capacidad para influir en la toma de las decisiones que las afectan.

Los servicios urbanos fueron objeto de una clara remercantilización. Los resultados son conocidos: concentración económica en las ciudades por aumento de las ganancias del capital y creciente peso económico en la reproducción de la población, desigualmente distribuido (CoInG, 2005; Hayata, 2010; Pírez, 2009).

\section{LOS SERVICIOS URBANOS EN LA URBANIZACIÓN POPULAR}

Las características del desarrollo capitalista subordinado de América Latina dejan fuera de las condiciones formales (de mercado o estatales) de acceso a los bienes urbanos a una parte importante de la población. Como consecuencia, se observan, por un lado, procesos

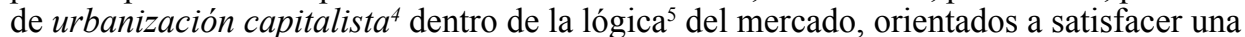
necesidad y a obtener una ganancia no inferior, por lo menos, a la media. Estos procesos se adecuan a las normas que regulan la propiedad del suelo y la producción, circulación y distribución de los bienes urbanos. Así, ofrecen suelo, vivienda, infraestructuras, servicios y equipamientos, como mercancías, a la población que puede pagarlos. Por otro lado, la urbanización popular orientada predominantemente a la satisfacción de la necesidad de bienes urbanos fuera del mercado y de la lógica política del Estado, que permite acceder al suelo en condiciones precarias (invasión de propiedad privada o estatal, compra a urbanizadores clandestinos) o no. Luego las familias producen, normalmente por autoconstrucción, las viviendas, infraestructuras (y servicios) y equipamientos. ${ }^{6}$ 
Mientras en la urbanización formal los servicios urbanos son un componente dado, en la urbanización popular son una carencia más. En este caso, la población tiende a demandar la provisión ante organismos estatales o a producir por sí misma (con mayor o menor apoyo estatal o social) algunos de esos servicios. Esto sucede no solamente en los inicios de la urbanización, sino que se prolonga en el tiempo, tal como ha sido mencionado en el trabajo pionero de Matos Mar para las barriadas de Lima (Matos MAR, 1967: 38), cuyas descripciones son análogas a las que ofrecen investigaciones realizadas en otras ciudades y en otros tiempos. ${ }^{7}$

La producción popular, como forma no capitalista organizada por la necesidad, es un proceso desmercantilizador que adopta varias formas: consumo por fuera de las relaciones mercantiles, en procesos informales que se concretan en conexiones clandestinas a las redes formales, particularmente de agua y de electricidad (Pírez, 2000: 62); producción fuera de las relaciones mercantiles, en procesos de autoconstrucción basados en el aporte casi exclusivo de recursos de los pobladores y, fundamentalmente, de su trabajo. En estos casos, la producción se organiza con predominio de la lógica de la necesidad, más allá de que algunos insumos sean producidos con la lógica de la ganancia (materiales, servicios, tal vez suelo). La desmercantilización de estos procesos no va acompañada de redistribución, por lo que tienden a conformar una situación de expoliación urbana (KOWARICK, 1981).

No todos los servicios de redes pueden ser sustituidos por procesos informales de producción, tanto por razones económicas como técnicas, pero existe una gran cantidad de casos en servicios de agua y saneamiento, energía eléctrica, gas y transporte de personas (Pírez, 2000).

Las tomas clandestinas de las redes formales de distribución de agua son posibles siempre que en la cercanía de los asentamientos existan esas redes. Cuando no es así, se encuentran desde modalidades que mantienen el carácter de bienes colectivos con organizaciones no mercantiles, como las cooperativas, que realizan perforaciones para obtener agua que distribuyen entre sus socios (BRUNSTEIN, 1988; MutUBERRÍA, 2007), hasta modalidades individuales, como perforaciones, generalmente familiares, donde existen napas subterráneas accesibles con cierta facilidad, o construcción de pozos sépticos en donde se arrojan las aguas utilizadas (Pírez, 2000).

En el caso de la electricidad, la producción popular es más compleja y por lo general adopta la forma de conexiones clandestinas (Pírez, 2009).

6- Ver Duhau, 1998: Matos Mar, 1967 y Schteingart, 2001 .

7- Ver Cuenya et ál., 1984; Cravino, 2006; Duhau, 1998; Schteingart, 2001. 
La producción individual implica un retroceso en la socialización de las condiciones de reproducción de la población: la necesidad, de agua, por ejemplo, vuelve a individualizarse con un conjunto de pérdidas: en la calidad del servicio con impacto en la salud; económicas por la aplicación de muchas horas de trabajo, además de los insumos y maquinarias que deben ser adquiridos mercantilmente, ambientales si contribuyen a la contaminación de las aguas subterráneas y superficiales.

En esos procesos intervienen diferentes actores: sectores populares que no consiguen los recursos suficientes para resolver en el mercado sus necesidades de asentamiento urbano; organizaciones estatales que se caracterizan por su omisión (falta de políticas) como por su acción (regulaciones que no pueden cumplirse en la producción popular de la ciudad) y actores capitalistas, que "hacen negocios" con el suelo de las urbanizaciones populares: principalmente fraccionadores (formales o no), propietarios de suelo urbano y diversos productores de los bienes necesarios para el servicio (SCHTEINGART, 2001: 91).

Las condiciones de exclusión que caracterizan la urbanización de la región (DuHAU, 1998; PrADILla, 1981 y 2009) dan pie a la capacidad estatal de controlar las demandas sociales, con amplio margen de discrecionalidad. Todo ello con bajo costo político y económico, ya que se estructuran "diferentes formas de intercambio cuya reproducción resulta facilitada y estimulada en situaciones en las que, como es el caso de los asentamientos populares, están en juego bienes tan básicos como la vivienda" (DuHAu, 1998: 15). Como resultado, los beneficios sociales son distribuidos entre el reconocimiento de derechos y las relaciones personalizadas de intercambio (clientelares), con cierta tendencia al predominio de estas.

Las proposiciones anteriores son particularmente aplicables a las condiciones imperantes entre 1940 y fines de los años 70. Corresponden al período de la industrialización por sustitución de importaciones, con intervención del Estado orientada a generar condiciones para el crecimiento económico, facilitando los procesos privados de acumulación. Si bien esas políticas definieron formalmente servicios urbanos orientados por su universalidad, los estados no lograron mantener el ritmo del crecimiento demográfico y territorial de las áreas urbanas. Correlativamente, los aparatos gubernamentales tendieron a tolerar las producciones clandestinas de la urbanización popular.

Esas acciones estatales, pese a su limitación, fueron puestas en cuestión a partir de los años 80. La crisis económica de la región y, particularmente, las políticas que se impusieron, dieron lugar a la transformación de las economías y los estados en procesos de reestructuración neoliberal, tal como mencionamos. El resultado fue el aumento de la urbanización 
popular. Por ejemplo, en el Área Metropolitana de Buenos Aires, entre 1981 y 1991, el 25 $\%$ de los nuevos habitantes se ubicaba en asentamientos informales, mientras que entre 2001 y 2006 lo hizo el 60 \% (CRAVINO ET ÁL. 2008: 105).

\section{LA GESTIÓN DE LOS SERVICIOS URBANOS: ENTRE LO TÉCNICO Y LO POLÍTICO}

En los años 80 los estudios sociales en América Latina tendieron a focalizarse sobre lo local. Esa atención estaba asociada, de alguna manera, a la descentralización que era conceptualizada entre dos extremos: aplicación de ajustes neoliberales y fragmentación de los conflictos; e intentos de democratización y de atención a las necesidades sociales. Con referencia a los servicios, se suponía que la descentralización "propiciaría una mayor eficacia en la prestación de los servicios públicos y sociales y, en consecuencia, una mejora sustancial de la calidad de vida de la población”" (Rodríguez y VeLÁzQUez 1994a: 19).

El "descubrimiento" de lo local y la postulación de la descentralización fueron parte de la reformulación de enfoques orientada tanto hacia recortes locales como coyunturales para la investigación social. En esa tarea, se utilizó el término gestión, referido a objetos de estudio que habían sido trabajados dentro del concepto más amplio de políticas urbanas o, de manera más abstracta aun, como parte de la relación entre el Estado y la urbanización.

Los análisis en el nivel local de gobierno introdujeron una mirada particular y, al mismo tiempo, una discusión sobre la noción de gestión urbana. La gestión de los servicios - componente de aquella - fue definida, por una parte, como un conjunto de "prácticas institucionales, administrativas y técnicas, públicas (preferentemente a nivel local) que conciernen a la creación y administración de los servicios urbanos"; y, por otra, como "las prácticas de gestión de la población en torno al aprovisionamiento y consumo de estos servicios" (COULOMB, 1993: 17-18). La gestión es "colocada" entre el ámbito gubernamental y la sociedad civil. Según Coulomb, ambas dimensiones son inseparables, y se dan en un continuo entre necesidad-demanda y respuesta desde los aparatos de gobierno local. La gestión se desenvuelve entre dos lógicas fundamentales: una política y otra técnica, aunque parece privilegiarse la lógica técnica, dada la definición de "prácticas institucionales, administrativas y técnicas".

En otra perspectiva, a partir de la noción de gobierno de la ciudad, se entiende la gestión como un "conjunto de componentes: desde las relaciones estrictamente politicas entre los actores de la ciudad y sus representantes, uno de cuyos resultados es la producción de normas que regulan acciones y relaciones en la ciudad, pasando por funciones eco-

8 - Conviene recordar que esos trabajos tuvieron un importante sesgo normativo. 
nómicas, como promoción y orientación de actividades, o sociales en la reproducción de la fuerza de trabajo" (Pírez, 1994: 54). Allí, la gestión urbana puede ser "el conjunto de procesos dirigidos a articular (utilizar, coordinar, organizar, asignar) recursos (humanos, financieros, técnicos, organizacionales, politicos, naturales) para generar las condiciones que permitan producir, hacer funcionar y mantener la ciudad, brindando a las actividades económicas y a la población los satisfactores para sus necesidades" (Pírez, 1994). Es de tal manera una actividad político-técnica: "parte de una política de gobierno e implica una orientación de procesos urbanos que supone decisiones sobre intereses, a la vez que depende de un adecuado manejo instrumental (técnico) para el logro de sus objetivos" (Pírez, 1994: 58).

Estudiar los servicios urbanos en esta perspectiva implica conocer "no solo la forma como la población satisface sus necesidades individuales y colectivas, sino también la manera como se está redefiniendo el rol del aparato público y sus relaciones con los distintos sectores de la sociedad” (RodríGuez y Velásquez, 1994b: 209).

Los actores sociales que participan en los procesos de gestión de los servicios establecen relaciones que consolidan modalidades particulares. Las formas que esas relaciones pueden adoptar, y que se configuran como modelos, dependen de los contextos institucionales y sociopolíticos. La diversidad de esos contextos explica la existencia de "varios modos de gestión, en los que el peso de los distintos actores y de sus respectivas racionalidades sería diferente", consolidando "formas de organización y manejo de los recursos... para definir, diseñar, ejecutar y evaluar los planes y programas relativos a la prestación de los servicios" (RODRíGUEZ y VelásQuez, 1994b: 213-214). Configuran unidades de significación en la organización de la gestión de los servicios y, por ende, en sus efectos urbanos y sociales.

Es posible clasificar esas modalidades atendiendo a las relaciones entre sus tres actores principales (estatales, empresas capitalistas y usuarios) e identificando tres dimensiones (nivel gubernamental implicado, relación entre sector estatal y sector privado y existencia de procesos participativos). Con esa base es posible identificar modelos entendidos como tipos ideales. Gestión estatal, cuando organizaciones gubernamentales tienen bajo su responsabilidad, y a su cargo directo, la totalidad de la prestación de un servicio, incluyendo su producción. Gestión privada si empresas capitalistas se hacen cargo de esa prestación, siendo responsables básicamente de la producción, pero también de fases correspondientes a su provisión. En este caso el servicio depende en su totalidad de "procedimientos de mercado", regulados por normas gubernamentales. Gestión mixta con control estatal cuando el aparato gubernamental mantiene el control de la provisión e incluye a empresas 
privadas, particularmente en algún momento de su producción, combinando organismos gubernamentales y empresas capitalistas, procedimientos gubernamentales y de mercado. Pueden darse también en forma centralizada o descentralizada. En los modelos descentralizados intervienen organismos gubernamentales o empresas públicas que corresponden a los niveles intermedio (provincias, estados o departamentos) o local (municipios), y que se hacen cargo en forma plena del servicio, son interlocutores de las empresas responsables o controlan el servicio que incorpora a empresas capitalistas (Pírez, 2000).

Trabajos posteriores definieron modelos de gestión de servicios en diferentes momentos históricos. El estudio de la distribución eléctrica en la Región Metropolitana de Buenos Aires (RMBA) encontró cuatro modalidades. Desde fines del siglo XIX hasta 1907 la distribución eléctrica fue una actividad económica privada sin ninguna regulación, como cualquier otra actividad económica capitalista. En 1907, el gobierno local logró regular la actividad de las empresas privadas, ejerciendo sobre ellas cierto control, configurando una modalidad privada-descentralizada. En los años 40 y 50, el gobierno federal estatizó las empresas privadas que prestaban el servicio eléctrico en toda la RMBA. Los gobiernos locales perdieron el control del servicio eléctrico, que pasó a organismos federales que regulan, producen, distribuyen y controlan, en una modalidad estatal-centralizada. En la década de 1990, como parte de la reestructuración neoliberal, el gobierno federal privatizó la empresa metropolitana de electricidad, conservando la atribución de regular y controlar el servicio. Se configuró una modalidad privada-centralizada (Pírez, 2000; Pírez, 2009).

El análisis histórico muestra la relevancia de esas diferentes modalidades, en la configuración y funcionamiento de la ciudad y, en especial, para conocer el lugar de la población de bajos recursos en el servicio (Pírez, 2009 y 2011).

\section{CONCLUSIONES}

A modo de conclusión, proponemos algunos elementos que pueden servir para construir un esquema para ser desarrollado teórica y empíricamente.

\section{Urbanización y servicios urbanos}

Los servicios urbanos son parte de las condiciones de reproducción de las formas urbanoindustriales capitalistas. Allí, cierta oferta de infraestructuras y servicios es necesaria para la reproducción de los capitales individuales y de la fuerza de trabajo. ${ }^{9}$ Sin embargo, esos bienes y servicios difícilmente son producidos dentro de los procesos capitalistas indivi- 
9- Una misma infraestructura o servicio puede contribuir a la reproducción del capital, como a la de la fuerza de trabajo y también a la de la población en general.

10- Es evidente que nos referimos a sociedades capiferimos
talistas. duales y, por lo mismo, son objeto de producciones alternativas. Una manera de analizar esa condición es referirla a la perspectiva de la mercantilización-desmercantilización, en el sentido de EsPing-ANDERSEN (1993) y OfFe (1990). La inclusión de esos bienes en la generalizada mercantilización que supone el capitalismo se vuelve contradictoria con el desarrollo del conjunto de los procesos de acumulación. En consecuencia, se configuran alternativas que significan procesos de desmercantilización. Esto es, formas de producción de los servicios que, de alguna manera y en cierto grado, desmercantilizan su acceso, es decir, permiten consumir esos bienes sin tener que contar con las cantidades monetarias que hacen falta en los procesos mercantiles.

En ese contexto estructural, los servicios urbanos son definidos por la relación, en cada sociedad $^{10}$, entre mercado de trabajo (incluyendo relaciones de distribución económicas), mercado de los bienes urbanos (suelo, vivienda, infraestructuras, servicios) y Estado (políticas públicas).

Los servicios urbanos son así componentes de la urbanización capitalista cuya significación depende de dos perspectivas: la del desarrollo capitalista en general y la del desarrollo capitalista subordinado. Además, su comprensión está relacionada con las distintas fases del desarrollo capitalista que - como hemos visto para América Latina- han sido analizadas en términos de desarrollo por sustitución de importaciones con intervención estatal y reestructuración neoliberal.

En las sociedades de capitalismo avanzado, con Estado de Bienestar, las alternativas desmercantilizadoras estuvieron, fundamentalmente, a cargo de los estados, que tuvieron así un papel central en la urbanización. La intervención estatal tendió a garantizar la mencionada desmercantilización, aplicando recursos fiscales e incluyendo situaciones de redistribución social vertical. Esa intervención compensó la dificultad de su producción individual. Sin embargo, este rasgo se mostró menos estructural que propio de una fase de desarrollo, como lo muestra la creciente participación de los capitales individuales en las últimas décadas. Esa participación privada está asociada a la generación de condiciones (de posibilidad) desde las políticas públicas, además de la aplicación de innovaciones tecnológicas y la conformación de fuertes conglomerados privados con gran capacidad financiera.

Diferente es la situación en el desarrollo capitalista subordinado de América Latina. La urbanización tiende a asociarse menos a la industrialización local que a la inserción en la división internacional del trabajo: territorios asociados a los procesos industriales de Europa o los Estados Unidos. En esa inserción, los procesos locales de acumulación de 
capital transfieren parte de su excedente a las economías centrales. Esto supone un freno para el desarrollo de las fuerzas productivas, implica bajo dinamismo en la acumulación de capital y una tendencia hacia la concentración de la producción industrial y su distribución desigual en el territorio. Los estados muestran limitada capacidad de captación económica (fiscal), asociada a la acumulación subordinada y a su debilidad política frente a los sectores económicamente poderosos, muchos de ellos de base internacional. Los mercados de fuerza de trabajo urbanos se caracterizan por la fuerte presencia de inserciones limitadas, precarias y mal remuneradas, en contextos de gran desigualdad de ingresos, con estructural subutilización de la mano de obra (JARAMillo, 1988; Pradilla, 1981; SzRETTER, 1990).

La consecuencia es que una porción importante de la población queda fuera de las formas capitalistas (mercantiles) de producción urbana, incluyendo infraestructuras y servicios. Esto significa la exclusión (absoluta o relativa) de esa población de los procedimientos formales: la producción urbana dentro del mercado, de acuerdo con las normas estatales que rigen la producción, circulación y consumo de esos bienes. Al mismo tiempo, frente a esa exclusión, se observan diferentes y numerosos procesos de producción de infraestructuras y servicios urbanos, junto a otros bienes urbanos, por fuera de aquella formalidad, organizados predominantemente por una lógica de necesidad.

Las condiciones particulares del desarrollo subordinado hacen emerger una situación que puede considerarse paradójica: urbanización sin servicios. Existen procesos de urbanización y áreas urbanas resultantes que no cuentan (de manera absoluta o relativa) con infraestructuras ni servicios urbanos. Para la población latinoamericana que queda fuera del mercado formal, el suelo urbano se produce y oсира (de manera formal o informal) sin que exista una oferta suficiente de infraestructuras. Esto significa una contradicción conceptual, frente a la noción convencional de la urbanización (construida a partir del desarrollo capitalista clásico y el Estado de Bienestar). Sin embargo, es coherente con los procesos históricos de ese desarrollo capitalista: buena parte de la población urbana se asienta en áreas urbanas que no cuentan con esas infraestructuras y que muestran un proceso largo en el tiempo para su producción, dado que infraestructuras y servicios vienen después, como consecuencia de acciones que llevan a cabo los pobladores.

Esas formas urbanas, las del hábitat popular o de la urbanización popular, implican una peculiar relación entre mercado de trabajo, mercado de bienes urbanos y políticas públicas, que se define por la existencia de acciones, normalmente colectivas, de producción socialmente desmercantilizada a cargo de la misma población necesitada, que aplica para ello, particularmente, su fuerza de trabajo. No solamente se accede así al suelo y se produce 
11- Puede verse, para el caso de Argentina, el trabajo de Torre y Pastoriza, en el cual se habla de procesos de "democratización del bienestar" y no Estado de Bienestar (Torre y Pastoriza, 2002). la vivienda, sino que también se producen por fuera de las condiciones formales algunos servicios urbanos de infraestructura. Esas experiencias suelen incluir vinculaciones con organismos gubernamentales, no gubernamentales o universitarios, que contribuyen a potenciar el esfuerzo social.

Las formas de producción no formales han sido objeto de más de una interpretación. Es posible identificar dos situaciones polares: la producción popular o autoproducción, como un instrumento de creatividad social y de satisfacción de necesidades o como un proceso de sobre-explotación.

\section{El papel del Estado}

El Estado cumple un papel central en la urbanización capitalista, que se relaciona con su contribución a la acumulación, en gran medida por medio de diferentes procesos de desmercantilización, y su contribución a la legitimidad en general. Las formas de Estado de Bienestar, en las que se reconocen derechos de ciudadanía que incluyen la satisfacción de necesidades de vivienda y asentamiento, significaron una consolidación de ese papel, más allá de sus contradicciones y de su decadencia en las dos últimas décadas. En estas, la reestructuración neoliberal modificó las políticas, disminuyendo su capacidad redistributiva y ampliando las oportunidades de valorización de los capitales individuales.

En las sociedades latinoamericanas, en las que difícilmente se pueda afirmar que se consolidaron propiamente relaciones de Estado de Bienestar ${ }^{11}$, el papel del Estado es también importante. Durante la fase desarrollista los estados latinoamericanos construyeron organizaciones que se hicieron cargo de la producción de infraestructuras y servicios, orientada formalmente con sentido de universalidad. Si bien esa orientación llevó principalmente a generar condiciones para el desarrollo de las actividades industriales que se promovían, se atendieron necesidades de infraestructura de la población. Esos esfuerzos no llegaron a cubrir al conjunto de la creciente población urbana, quedando buena parte de las familias de bajos ingresos libradas a su suerte. Consecuentemente, una porción importante de los territorios urbanos, en particular en las áreas metropolitanas, crecieron sin esos bienes urbanos. En estos casos, esa producción dependió de las acciones de la población que se asentaba, consolidando las diferencias socioeconómicas en la producción de territorios urbanos desiguales.

Ante esa incapacidad estatal, los gobiernos se mostraron permisivos frente a las clandestinidades de la producción no formal: así se descargaban —en parte al menos- de su responsabilidad, y establecían relaciones políticamente desiguales, personalizadas con 
la población necesitada, en la distribución de diferente recursos: además de la permisividad, apoyos materiales, incluyendo transferencias financieras. La dimensión política de la relación entre los organismos gubernamentales y la población excluida del mercado formal ha sido un elemento clave para la estructuración de las relaciones de dominación en las ciudades de la región. En la producción de estos bienes urbanos dos actores son fundamentales: organismos estatales y sectores populares.

Con la reestructuración neoliberal, el papel de los estados tuvo una fuerte transformación. Más allá de cambios particulares, es necesario comprender que los aparatos estatales modificaron globalmente la orientación de sus intervenciones: desde cierto sentido de bienestar y de legitimización, hacia el fortalecimiento de la acumulación y la dominación. En consecuencia, no solamente disminuyeron los recursos destinados a la producción de bienes urbanos, sino que esto sucedió de manera especial en la atención de las necesidades de la población de menores recursos. La producción de infraestructuras y de servicios urbanos fue uno de los aspectos centrales de las transformaciones: desregulación, competencia y privatizaciones fueron los objetivos que se propusieron, pasando de la permisividad al disciplinamiento del mercado. La simultánea modificación de los tres componentes centrales para el análisis alteró la significación de esos servicios: exclusiones del mercado de trabajo e incrementos en la concentración económica, disminución de las políticas sociales gubernamentales y aumento del peso económico de los bienes urbanos en los presupuestos de las familias, particularmente de aquellas de menores recursos. Detrás de esos cambios se encuentran las transformaciones en las regulaciones que, más que desregulación en sentido lato, significaron modificaciones en la orientación preexistente. Como consecuencia, junto a los organismos estatales y los sectores populares aparecen actores económicos privados capitalistas con una gran capacidad de decisión para orientar las relaciones que definen a los servicios.

Esos cambios fueron percibidos en sus diferentes combinaciones de relaciones entre los actores y su consolidación en modelos o modalidades de gestión. Se diferenciaron claramente dos dimensiones principales: privado/estatal y centralizado/descentralizado. La tendencia central fue el predominio de formas privadas, en términos de (re) mercantilización de esos sectores, en algunos casos en procesos de descentralización (Brasil, Colombia), mientras que en otros con base en los niveles centrales (Argentina, Chile).

En estos procesos se identificó la relevancia de los marcos institucionales de regulación y control. Tal fue esa consideración que se supuso que lo importante no era ya la propiedad o control económico de los productores-prestadores de servicios, sino las regulaciones 
que ordenaban sus actividades y sus relaciones con los usuarios. Pero al mismo tiempo, y en particular con base en los estudios de la privatizaciones en Buenos Aires, se observó la capacidad de las nuevas empresas privadas de modificar esas regulaciones en su favor, y de condicionar el papel de los organismos de control, dando lugar a la formación de "ámbitos privilegiados de acumulación" que, con base en decisiones institucionales (no de mercado) permitieron la obtención de ganancias extraordinarias y, a la vez, subordinaron a los usuarios, en particular a las familias de menores recursos. Pero no solamente se perjudicaron los usuarios residenciales, sino que esas condiciones institucionales debilitaron las condiciones de acumulación del resto de las actividades y así, al proceso global de acumulación.

La reestructuración neoliberal mostró, en consecuencia, una fuerte politización en la gestión de los servicios urbanos, no ya en favor de los sectores populares, sino de las empresas: de la permisividad al disciplinamiento para aquellos, mientras que nueva permisividad para estas.

Esos procesos significaron una remercantilización de la producción de infraestructuras y servicios urbanos que puso relativamente en riesgo la tasa de ganancia global y debilitó fuertemente las condiciones de reproducción de la población.

\section{BIBLIOGRAFÍA}

ASPIAZU, Daniel (compilador) (2002) Privatizaciones y poder económico. La consolidación de una sociedad excluyente. Universidad Nacional de Quilmes, Bernal.

BRUNSTEIN, Fernando (1988). "Saneamiento hídrico en el Gran Buenos Aires. Límite de la precariedad". En: Brunstein, Fernando (comp.) Crisis y servicios públicos. Agua y saneamiento en la región metropolitana de Buenos Aires. Cuadernos del CEUR 23, Centro de Estudios Urbanos y Regionales, Buenos Aires.

CASTELLS, Manuel (1995) La ciudad informacional. Tecnologías de la información, reestructuración económica y el proceso urbano. Alianza Editorial, Madrid.

COING, Henri (2005). "Servicios públicos en Bogotá. Impacto de las reformas sobre la solidaridad territorial y social". En: Gouëset, Vincent, Luis Mauricio Cuervo, Thierry Lulle y Henri Coing (coordinadores): Hacer Metrópoli. La región urbana de Bogotá de cara al siglo XXI. Universidad Externado de Colombia, Bogotá.

COULOMB, René (1993). "La participación de la población en la gestión de los servicios urbanos: ¿Privatización o socialización?”. En: Azuela, Antonio y Emilio Duhau (Coordi- 
nadores) Gestión urbana y cambio institucional. Universidad Autónoma Metropolitana, Azcapotzalco.

CRAVINO, María Cristina (2006) Las villas de la ciudad. Mercado e informalidad urbana. UNGS, Los Polvorines.

CRAVINO, María Cristina; DEL RÍO, Juan Pablo y DUARTE, Juan Ignacio (2008).

"Un acercamiento a la dimensión cuantitativa de los asentamientos y villas del Área Metropolitana de Buenos Aires". En: Cravino, María Cristina (organizadora). Los mil barrios (in)formales. Aportes para la construcción de un observatorio del hábitat popular del Área Metropolitana de Buenos Aires. UNGS, Los Polvorines

CUENYA, Beatriz; PASTRANA, Ernesto y YUJNOVSKY, Oscar (1984) De la villa miseria al barrio autoconstruido. Ediciones CEUR, Buenos Aires.

CUERVO, Luis Mauricio (1997). "El agua potable como bien mayor". Revista Regulación No. 2, agosto de 1997, Bogotá, p. 135-160.

CUERVO, Luis Mauricio (1998). "El régimen de servicios públicos domiciliarios. Primer Tiempo: Tecnocracias $=1$, Autonomía local $=0$ ". En: La investigación regional y urbana en Colombia. Desarrollo y territorio 1993-1997. Tomo 1, Carlos Valencia Editores, Bogotá

DUHAU, Emilio (1998) Hábitat popular y política urbana. Miguel Ángel Porrúa -UAM-A, México.

ESPING-ANDERSEN, G. (1993) Los tres mundos del Estado del Bienestar. Valencia: Edicions Alfons El Magnànim-Generalitat Valenciana-Diputació Provincial de València. HARVEY, David (2007) A Brief History of Neoliberalism. Oxford University Press, New York.

HAYATA, Noriko (2010) La ilusión de la participación comunitaria. Lucha y negociación en los barrios irregulares de Bogotá 1992-2003. Bogotá: Universidad del Externado. 478p. JARAMILLO, Samuel (1988). "Crisis de los medios de consumo colectivo urbano y capitalismo periférico". En: Cuervo, Luis Mauricio, Samuel Jaramillo, Jorge Iván González y Fernando Rojas Economía politica de los servicios públicos. Una visión alternativa. CIDEP, Bogotá.

KOWARICK, Lucio (1981). "El precio del progreso: crecimiento económico, expoliación urbana y la cuestión del medio ambiente". En: Sunkel, O. y N. Gligo, Estilos de desarrollo y medio ambiente en América Latina. El Trimestre Económico, México.

MATOS MAR, José (1967) Estudio de las barriadas limeñas. Universidad Nacional Mayor de San Marcos, Lima.

MURILLO, M. Victoria y Diego FINCHELSTEIN (2004). "Privatización y poder de mercado: el caso de la generación de energía eléctrica en la Argentina”. En: Desarrollo Económico, Vol. 44m No. 173, pp. 131-144. 
MUTUBERRÍA, Valeria (2007) Los servicios públicos urbanos como medios colectivos para la producción y reproducción de la vida de los sujetos en sociedad desde la perspectiva de la economía social. Análisis de experiencias de gestión colectiva en el Gran Buenos Aires. Tesis de Maestría en Economía Social, Universidad Nacional de General Sarmiento, Buenos Aires.

OFFE, C. (1990) Las contradicciones del Estado del Bienestar. Madrid: Alianza.

PÍREZ, Pedro (1994) Buenos Aires metropolitana. Política y gestión de la ciudad. Centro Editor de América Latina, Buenos Aires.

PÍREZ, Pedro (1995). “Actores sociales y gestión de la ciudad". En: Ciudades, año 7, No. 28, octubre-diciembre 1995.

PÍREZ, Pedro (2000). "Servicios urbanos y equidad en América Latina. Un panorama con base en algunos casos", Serie Medio Ambiente y Desarrollo No. 26, CEPAL, Santiago de Chile.

PIREZ, Pedro (2009) Las sombras de la luz. Distribución eléctrica, configuración urbana y pobreza en la Región Metropolitana de Buenos Aires. EUDEBA, Buenos Aires.

PÍREZ, Pedro (2011). "Distribución eléctrica y desigualdades en la urbanización en América Latina". En: Seminario Internacional Infraestructuras Urbanas en América Latina: gestión y construcción de servicios y obra pública. FLACSO, Quito.

PRADILLA, Emilio (1981). "Desarrollo capitalista dependiente y proceso de urbanización en América Latina". Revista Interamericana de Planificación, Vol. XV, No. 57, pp. 73-99. PRADILLA, Emilio (2009). “Acumulación de capital y estructura territorial en América Latina: teoría e historia". En: Pradilla, Emilio, Los territorios del neoliberalismo en América Latina. Compilación de ensayos. UAM-Porrúa, México.

RODRÍGUEZ, Alfredo y VELÁSQUEZ, Fabio (1994a) “Presentación” en Rodríguez, Alfredo y Fabio Velásquez (editores). Municipio y servicios públicos. Gobiernos locales en ciudades intermedias de América Latina. Ediciones Sur, Santiago de Chile.

RODRÍGUEZ, Alfredo y VELÁSQUEZ, Fabio (1994b). “Introducción” Segunda Parte en Rodríguez, Alfredo y Fabio Velásquez (editores). Municipio y servicios públicos. Gobiernos locales en ciudades intermedias de América Latina. Ediciones Sur, Santiago de Chile.

RODRÍGUEZ, María Carla et ál. (2007) Políticas del hábitat, desigualdad y segregación socioespacial en el área metropolitana de Buenos Aires, AEU-IIGG/FSOC-UBA, Buenos Aires.

SCHTEINGART, Martha (2001) Los productores del espacio habitable. Estado, empresa y sociedad en la Ciudad de México. El Colegio de México, México.

TORRE, Juan Carlos y PASTORIZA, Elisa (2002). "La democratización del bienestar". En: Torre, Juan Carlos (Director). Los años peronistas (1943-1955): 257-312. Sudamericana, Buenos Aires. 